\title{
Fibrosarcoma in Pediatric Patients: Results of the Italian Cooperative Group Studies (1979-1995)
}

\author{
GIOVANNI CECCHETTO, MD, ${ }^{1} *$ MODESTO CARLI, MD, ${ }^{2}$ RITA ALAGGIO, MD, ${ }^{3}$ PATRIZIA DALL'IGNA, MD, ${ }^{1}$ \\ GIANNI BISOGNO, MD, ${ }^{2}$ GIOVANNI SCARZELLO, MD, ${ }^{4}$ ILARIA ZANETTI, PhD, ${ }^{2}$ GIANFRANCO DURANTE, ${ }^{2},{ }^{5}$ \\ ALESSANDRO INSERRA, MD, ${ }^{6}$ FORTUNATO SIRACUSA, MD, ${ }^{7}$ AND MAURIZIO GUGLIELMI, MD ${ }^{1}$ \\ ${ }^{1}$ Department of Pediatrics, Division of Pediatric Surgery, University of Padua, Padua, Italy \\ ${ }^{2}$ Department of Pediatrics, Division of Onco-Hematology, University of Padua, Padua, Italy \\ ${ }^{3}$ Department of Oncologic Sciences-Pathology Unit, University of Padua, Padua, Italy \\ ${ }^{4}$ Division of Radiation Therapy, General Hospital of Padua, Padua, Italy \\ ${ }^{5}$ Division of Pediatric Surgery, Pediatric Hospital "C.Arrigo", Alessandria, Italy \\ ${ }^{6}$ Division of Pediatric Surgery, Pediatric Hospital "Bambin Gesù", Rome, Italy \\ ${ }^{7}$ Department of Pediatric Surgery, Istituto Materno Infantile, Palermo, Italy
}

Background and Objectives: Fibrosarcoma is a rare soft tissue sarcoma (STS) that has two peaks of incidence in pediatric patients: one in infants and young childern (infantile fibrosarcoma), another in older children ("adult type" fibrosarcoma). The purpose of this study was to describe the clinical features and the treatment results in patients affected by fibrosarcoma enrolled in two consecutive studies run by the STS-Italian Cooperative Group (ICG) between 1979 and 1995.

Patients and Methods: Twenty-five evaluable patients were grouped according the intergroup rhabdomyosarcoma staging (IRS) system: $12 \mathrm{Gr}$ I, $5 \mathrm{Gr}$ II, $8 \mathrm{Gr}$ III. The cut-off point between the two forms was considered the age of 2 years: 14 patients were affected by infantile and 11 by adult type of fibrosarcoma. The therapeutic guidelines were not strict and not different for the two forms: patients with initial macroscopic excision $(\mathrm{Gr}$ I-II) were given IVA or VAC; Gr III had VAC or VAIA and subsequent excision, if feasible. Radiation therapy (RT) was delivered in patients $>3$ years with microscopic (42 Gy) and macroscopic (54 Gy) residuals.

Results: Thirteen patients are alive without evidence of disease: 10 in first complete remission (CR), 7 Gr I, 1 Gr II, 2 Gr III, 3 in second CR after local relapse (LR) (2 Gr I, 1 Gr II). Two Gr I patients are alive with metastatic relapse. Nine patients died: six of progressive disease (PD) (three Gr II, three Gr III), two because of a second tumor (one Gr I, one Gr III) and one because of other causes (Gr III). One Gr III patient was lost at follow up, 2 years from diagnosis. The 10 years overall survival (OS) is $67.4 \%$ and the progression-free survival (PFS) is $52.2 \%$. The OS for patients $<2$ years is $78.6 \%$ ( 11 of 14 ), versus $51 \%$ for patients $>2$ years (5 of 11).

Conclusions: The complete excision at diagnosis was the treatment of choice and was related to the best outcome. Microscopical residuals were difficult to treat with chemo-radiotherapy in both forms of fibrosarcoma. Neoadjuvant chemotherapy (CT) obtained a partial remission (PR) only in three of eight cases, while no conclusions concerning the efficacy of CT for infantile forms are possible. Patients $<2$ years had a better outcome than

Contract grant sponsor: Consiglio Nazionale Delle Ricerche; Contract grant sponsor: Progetto Finalizzato; Contract grant number: CNR ACRO n.96.00658.PF39; Contract grant sponsor: MURST.
*Correspondence to: Giovanni Cecchetto, MD, Department of Pediatrics, Division of Pediatric Surgery, University of Padua, Via Giustiniani 3, 35128 Padua, Italy. E-mail: cecchett@child.pedi.unipd.it Accepted 23 August 2001 
the older ones: most of them had a tumor on extremities which was excised at diagnosis.

J. Surg. Oncol. 2001;78:225-231. (C) 2001 Wiley-Liss, Inc.

KEY WoRDS: fibrosarcoma; infantile fibrosarcoma; soft tissue sarcoma

\section{INTRODUCTION}

Fibrosarcoma represents about $10 \%$ of pediatric nonrhabdo soft tissue sarcomas (NRSTS) and is the most common soft tissue sarcoma (STS) in children under 1 year of age. There are two peaks of incidence: one in infants and young children (infantile fibrosarcoma) and another in patients aged between 10 and 15 [1,2].

Both are composed of spindle cells with different growth patterns: solid, hemangiopericitoma like, and herringbone. High mitotic index and high cellularity are present in spite of the low aggressivity of the lesion. An adequate immunohistochemical panel is necessary for a differential diagnosis with other sarcomas (malignant peripheral nerve sheat tumors, rhabdomyosarcomas, and smooth muscle tumors). A differential diagnosis with miofibromatosis and infantile hemangiopericytoma is sometimes impossible, even if a diffusely infiltrative growth pattern is more indicative for miofibromatosis [3]. The clinical characteristics of the tumor, the age of the patient, and presently also cytogenetic differences, are considered main factors in distinguishing the infantile or the adult type and deciding on their adequate treatment $[4,5]$. The age limit between the two forms is a matter of discussion: at present most authors consider as cutoff point the age of 2 years, others the age of 4 or 5 years [3]. Surgery is the mainstay of treatment in both forms; the role of chemotherapy (CT) and radiation therapy (RT) is still debated, even if the infantile fibrosarcoma seems to be more chemosensitive.

This report reviews the clinical characteristics and the treatment results of children and adolescents with fibrosarcoma, registered in the studies of the STS-Italian Cooperative Group (ICG).

\section{PATIENTS AND METHODS}

Between January 1979 and December 1995, 30 patients under 18 years affected by fibrosarcoma were registered in two consecutive national protocols RMS-79 and RMS-88. All the cases had a localized disease. In this paper, we describe the 25 of 30 patients, 10 of the first study and 15 of the second, for which adequate data were available. They were 10 males and 15 females with a median age of 24 months (range 8 days-187 months) representing $12 \%$ of patients with NRSTS (25 of 209) and $4.4 \%$ of those with STS (25 of 575) enrolled in the studies. All the histologic specimens were reviewed by a panel of pathologists. For all cases from a minimum of two to a maximum of eight Ematossilin-Eosin stained sections were reviewed and a complete immunohistochemical panel represented by S-100 (DAKO, Clone COW S100 diluition 1:7000), MNF116 (DAKO, Clone MNF116 diluition 1:100), HHF 35 (DBA Clone HHF35 diluition 1:300) was performed on formalin fixed, paraffin embedded specimens for differential diagnosis from other sarcomas. Diagnosis of fibrosarcoma was made when at the E.E. stain a highly cellular mitotically active neoplasia with spindle cells, without signs of neural or rhabdomioblastic differentiation was present and the himmunohistochemistry showed diffuse negativity for all markers (Fig. 1A,B). Only in one case a slight positivity for muscle Specific Actin (HHF 35) was evident. The age was the main criterium to differentiate infantile subtypes from adult subtypes: the fibrosarcomas found in patients younger than 2 years were considered infantile.

The group included 14 patients with the infantile form (in 7 of 14 under 3 months of age the tumor was considered congenital) and 11 with the "adult type" form. They were grouped according to the intergroup rhabdomyosarcoma staging (IRS) system: Gr I complete microscopical excision; Gr II excision with microscopical residuals (IIa) or with lymphnodes involvement (IIb) or both (IIc); Gr III biopsy or excision with macroscopical residuals. According to TNM pretreatment classification, tumors confined to the organ or tissue of origin were considered $\mathrm{T} 1$; those involving contiguous organs or structures $\mathrm{T} 2$.

The therapeutic guidelines of the studies were not strict, in particular there were no sharp differences in treatment between the infantile and adult forms of fibrosarcoma. Wide excisions were always recommended, and a more aggressive multidisciplinary approach was suggested in older patients. However, initial anatomic or funtional demolitive procedures (i.e., extremity amputation, pneumonectomy, large head-neck dissection, pelvic exenteration, procedures with permanent urinary or intestinal diversion) were discouraged. CT with vincristine, dactinomycin, and cyclophosphamide, or vincristine adriamycine and cyclophosphamide (VAC/CAV regimens, RMS-79), or ifosfamide, vincristine, dactinomycine (IVA regimen, RMS-88) was recommended for all the patients in Gr I and Gr II after primary surgery. Neoadjuvant CT with VAC regimen (cyclophosphamide, 


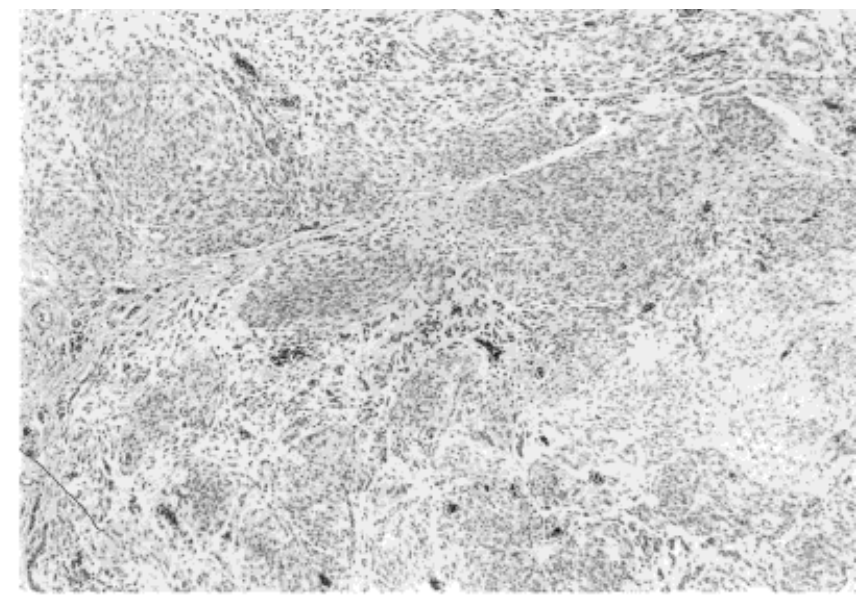

A

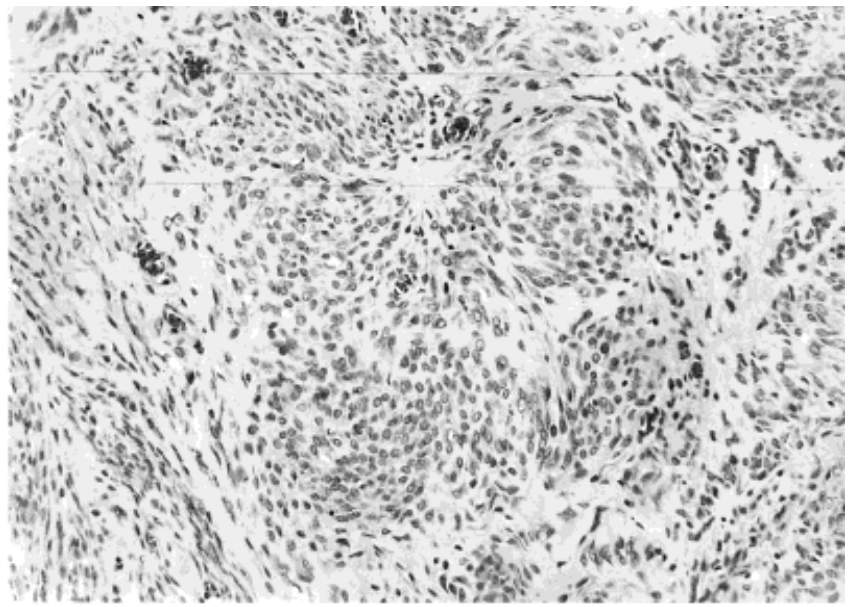

B

Fig. 1. Infantile fibrosarcoma with a solid growth pattern and collagen formation. Numerous dilated blood vessels are present (45 $\times$, EE stain) (A). Focal hemangiopericytoma-like pattern and whorling of cells resemble infantile myofibromatosis $(120 \times$, EE stain) $(\mathbf{B})$.

dactinomycin, vincristine) in the first study or with VAIA (vincristine, dactinomycin, ifosfamide, adriamycine) in the second study was given to patients in Gr III: a delayed surgery, if feasible, followed CT, and subsequently a maintenance $\mathrm{CT}$ with CAV/VAC or IVA was administered. RT was recommended in patients $>3$ years of age: 40-45 Gy in cases with microscopical disease after primary surgery, 45-54 Gy when delayed surgery was not radical or not possible. Children younger than 3 years did not receive RT because of the high risk of late sequelae. In the case of failure of CT and/or RT, mutilating surgery was accepted. Response to treatment was defined as follows: complete remission (CR) equals no evidence of disease; partial remission (PR) equals reduction $>50 \%$ of initial tumor volume; objective remission (OR) equals reduction $>25 \%$ of initial tumor volume; stable disease (SD) equals reduction $<25 \%$ of initial tumor volume; progression of disease (PD) equals increase of the initial tumor volume or appearance of new lesions.

\section{RESULTS}

The clinical features, the IRS Groups, the treatment and the outcome of the patients are reported in Table I. The extremities were the most frequent localizations (14 of 25), especially in patients under 2 years of age (10 of 14 , and 5 of 7 among the congenital cases). The masses in young patients had a fast growth in most instances. No significant differences were evident between patients younger and older than 2 years regarding the initial $\mathrm{T}$ status and the tumor size (Table II). Involvement of regional lymphnodes was not detected in any case at clinical and imaging investigations.
After initial surgery 12 patients were classified in Gr I, 5 in Gr II, (4 IIa, 1 IIc), and 8 in Gr III. Eight out of the 14 patients aged $<2$ years ( 4 of 7 patients among those with congenital fibrosarcoma) had initially a radical excision, while only 4 of 11 of the older patients had completely resected tumors at diagnosis. Three patients $<2$ years and two $>2$ years of age had microscopical residuals after initial surgery; a biopsy was performed initially in three children $<2$ years and in five $>2$ years.

The treatment and the outcome of the patients are described according to IRS Group:

Gr I: Five patients (aged 1, 3, 6, 27 months) had surgery alone; six (aged 1, 7, 17, 19, 101, 112 months) received surgery (mutilating in the oldest patient), and CT; a 177-month-old boy underwent the complete excision plus CT (IVA) and RT (40 Gy).

Eight cases achieved and mainained the first CR, but one, whose primary was on extremity, died of a second tumor (PNET) that occurred on the thoracic wall 3 years after the diagnosis of fibrosarcoma. The panel of pathologists confirmed two different tumors.

A local relapse (LR) occurred at 6, 8, and 10 months from diagnosis in three children, who had had surgery alone (two) and surgery + CT (one). After LR they received multimodal therapy which included mutilating surgery (amputation) in the first two: at present two are alive in second $\mathrm{CR}$ and one is alive with pulmonary metastases.

A 101-month-old girl treated with excision and CT suffered from pulmonary metastases at 58 months from diagnosis and is alive with disease.

Gr II: Four patients had a primary excision with microscopical residuals (Gr IIa). Two of them, aged 3 and 
TABLE I. Features, Treatment, and Outcome of the 25 Patients With Fibrosarcoma

\begin{tabular}{|c|c|c|c|c|c|c|c|}
\hline & $\begin{array}{l}\text { Age } \\
\text { mo/sex }\end{array}$ & Primary site & $\mathrm{T}$ status & IRS group & $\begin{array}{c}\text { Treatment } \\
\text { (CT response) }\end{array}$ & $\begin{array}{l}\text { Outcome and further } \\
\text { therapy }\end{array}$ & $\begin{array}{l}\text { Present status \& } \\
\quad \text { follow-up }\end{array}$ \\
\hline 1 & $1 / \mathrm{M}$ & Calf & T1a & III & CT (NE) & Cardiac malformation & Died 1 mo \\
\hline 2 & $1 / \mathrm{M}$ & Foot & $\mathrm{T} 1 \mathrm{~b}$ & I & $\mathrm{S}$ & LR (CT + Mut.S) & NED $120 \mathrm{mo}$ \\
\hline 3 & $1 / \mathrm{M}$ & Calf & T1a & I & S & & NED $104 \mathrm{mo}$ \\
\hline 4 & $1 / \mathrm{F}$ & Forearm & T1a & I & $\mathrm{S}+\mathrm{CT}$ & & NED 109 mo \\
\hline 5 & $2 / \mathrm{F}$ & Paravertebral & $\mathrm{T} 2 \mathrm{~b}$ & III & $\mathrm{CT}$ & PD & DOD $3 \mathrm{mo}$ \\
\hline 6 & 3/M & Retroperitoneal & $\mathrm{T} 2 \mathrm{~b}$ & I & $\mathrm{S}$ & & NED 197 mo \\
\hline 7 & $3 / \mathrm{F}$ & Arm & $\mathrm{T} 2 \mathrm{a}$ & IIa & S & & NED $98 \mathrm{mo}$ \\
\hline 8 & $6 / \mathrm{M}$ & Foot & T1a & I & $\mathrm{S}$ & & NED $60 \mathrm{mo}$ \\
\hline 9 & $7 / \mathrm{F}$ & Retroperitoneal & $\mathrm{T} 2 \mathrm{~b}$ & I & $\mathrm{S}+\mathrm{CT}$ & & NED $217 \mathrm{mo}$ \\
\hline 10 & $7 / \mathrm{F}$ & Arm & T1a & IIa & $\mathrm{S}$ & $\mathrm{LR}(\mathrm{CT}+\mathrm{S})$ & NED 109 mo \\
\hline 11 & $7 / F$ & Thigh & $\mathrm{T} 2 \mathrm{~b}$ & III & $\mathrm{CT}(\mathrm{OR})+\mathrm{S}$ & & NED $80 \mathrm{mo}$ \\
\hline 12 & $17 / \mathrm{F}$ & Buttock & $\mathrm{T} 1 \mathrm{~b}$ & I & $\mathrm{S}+\mathrm{CT}$ & $\mathrm{LR}(\mathrm{CT}+\mathrm{RT}+\mathrm{S})$ & NED 110 mo \\
\hline 13 & $19 / \mathrm{F}$ & Arm & $\mathrm{T} 1 \mathrm{~b}$ & I & $\mathrm{S}+\mathrm{CT}$ & & NED $186 \mathrm{mo}$ \\
\hline 14 & $24 / \mathrm{M}$ & Thoracic wall & $\mathrm{T} 2 \mathrm{a}$ & IIa & $\mathrm{S}+\mathrm{CT}$ & $\mathrm{LR}+\operatorname{Met}(\mathrm{CT}+\mathrm{S})$ & DOD 13 mo \\
\hline 15 & $27 / \mathrm{F}$ & Forearm & T1a & I & $\mathrm{S}$ & $\begin{array}{l}\text { Multiple LR } \\
\quad(\mathrm{CT}+\mathrm{RT}+\text { Mut.S })\end{array}$ & $\begin{array}{c}\text { AWD } 65 \text { mo, lung } \\
\text { metastases }\end{array}$ \\
\hline 16 & $51 / \mathrm{F}$ & Pericardial & $\mathrm{T} 1 \mathrm{~b}$ & III & $\mathrm{CT}(\mathrm{PD})+\mathrm{RT}$ & PD & DOD $7 \mathrm{mo}$ \\
\hline 17 & $60 / \mathrm{F}$ & Thoracic & $\mathrm{T} 1 \mathrm{~b}$ & III & $\mathrm{CT}(\mathrm{SD})+\mathrm{RT}+\mathrm{S}$ & & NED 175 mo \\
\hline 18 & $87 / \mathrm{M}$ & HNnPM & T1a & IIc & $\mathrm{S}+\mathrm{CT}+\mathrm{RT}$ & Lung metastases & DOD 24 mo \\
\hline 19 & $101 / \mathrm{F}$ & Thigh & $\mathrm{T} 1 \mathrm{~b}$ & I & $\mathrm{S}+\mathrm{CT}$ & Lung metastases & AWD 96 mo \\
\hline 20 & $112 / \mathrm{M}$ & Calf & $\mathrm{T} 1 \mathrm{~b}$ & I & Mut.S + CT & & $\begin{array}{l}\text { NED } 36 \text { mo, died } \\
\text { 2nd tumor }\end{array}$ \\
\hline 21 & $130 / \mathrm{F}$ & Pericardial & $\mathrm{T} 2 \mathrm{~b}$ & IIa & $\mathrm{S}+\mathrm{CT}$ & LR & DOD $10 \mathrm{mo}$ \\
\hline 22 & $156 / \mathrm{M}$ & Presacral & $\mathrm{T} 2 \mathrm{~b}$ & III & $\mathrm{CT}(\mathrm{PR})+\mathrm{RT}$ & Lung metastases & DOD 12 mo \\
\hline 23 & $167 / \mathrm{F}$ & Thigh & $\mathrm{T} 2 \mathrm{a}$ & III & CT $(\mathrm{PR})+$ Mut.S & & $\begin{array}{l}\text { NED } 132 \text { mo, died } \\
\text { 2nd tumor }\end{array}$ \\
\hline 24 & $177 / \mathrm{M}$ & Thoracic wall & T1a & I & $\mathrm{S}+\mathrm{CT}+\mathrm{RT}$ & & NED $71 \mathrm{mo}$ \\
\hline 25 & $187 / \mathrm{F}$ & HNPM & $\mathrm{T} 2 \mathrm{~b}$ & III & $\mathrm{CT}(\mathrm{PR})+\mathrm{RT}$ & & $\begin{array}{l}\text { NED } 24 \text { mo, lost } \\
\text { follow up }\end{array}$ \\
\hline
\end{tabular}

AWD, alive with disease; CT, chemotherapy; DOD, died of disease; Mut.S, mutilating surgery; LR, local relapse; NE, not evaluable; NED, no evidence of disease; OR, objective response; PD, progression of disease; PR, partial response; RT, radiotherapy; S, surgery; SD, stable disease; mo, month.

7 months, did not receive postoperative CT: the first is alive in CR, the second had a LR after 1 year and at present he is alive in second $\mathrm{CR}$ after a new radical excision. Two other patients, aged 4 and 24 months, who

TABLE II. Clinical Features and Surgical Groups According to Age

\begin{tabular}{lccc}
\hline & $\leq 2$ Years & $>2$ years & Total \\
\cline { 2 - 4 } No. & 14 & 11 & 25 \\
\hline Site & & & \\
$\quad$ Extremity & 10 & 4 & 14 \\
$\quad$ Axial & 4 & 7 & 11 \\
T status & & & \\
T1 & 9 & 7 & 16 \\
T2 & 5 & 4 & 9 \\
Size & & & \\
$\quad<5$ cm & 6 & 4 & 10 \\
$>5$ cm & 8 & 7 & 15 \\
IRS group & & & \\
I & 8 & 4 & 5 \\
II & 3 & 2 & 8 \\
III & 3 & 5 & \\
\hline
\end{tabular}

had CT after the excision, presented LR after 5 and 6 months, and died of progressive disease (PD) despite further multimodal therapy.

One patient in Gr IIc, an 87-month-old boy, presented pulmonary metastases after CT (VAC) and RT (50 Gy) and died of disease (DOD).

Gr III: Eight patients (aged 1, 2, 7, 51, 60, 156, 167, 187 months) with inoperable tumor underwent an open biopsy at diagnosis. Among the five patients treated in the RMS-79 study with neoadjuvant CAV regimen, three achieved a PR, while two showed a PD. Of the three patients treated in the second study with VAIA regimen, one obtained a minor response (OR), one had $\mathrm{SD}$ and one died during treatment because of cardiac malformation.

After neoadjuvant CT the seven patients were treated with radical surgery (two), incomplete excision $+\mathrm{RT}$ (one), RT + CT (three) and with CT alone (one). Four of the seven cases achieved the $\mathrm{CR}$, but one died because of a second tumor (not otherwise specified) that appeared 11 years later. Three patients never achieved the CR and died from PD. 
TABLE III. Overall Survival at 10 Years According to Age, IRS Group, Site, and Protocol

\begin{tabular}{lcccc}
\hline & $\begin{array}{c}\text { No. of } \\
\text { patients }\end{array}$ & Events & $\%($ CI 95\%) & $P$-value \\
\hline Age & & & & \\
$\quad<2$ years & 14 & 3 & $78.6(57.0-100)$ & 0.3 \\
$\quad>2$ years & 11 & 6 & $51.0(20.0-82.0)$ & \\
IRS group & & & & \\
$\quad$ I & 12 & 1 & $91.7(76.0-100)$ & 0.01 \\
$\quad$ II and III & 13 & 8 & $44.0(16.0-72.0)$ & \\
Site & & & & \\
$\quad$ Extremity & 14 & 3 & $85.7(67.4-100)$ & 0.08 \\
$\quad$ Axial & 11 & 6 & $42.4(11.8-73.0)$ & \\
Protocol & & & & \\
$\quad$ RMS-79 & 10 & 6 & $46.7(14.2-79.2)$ & 0.059 \\
$\quad$ RMS-88 & 15 & 3 & $80.0(60.0-100)$ & \\
\hline
\end{tabular}

\section{Present Status}

Thirteen patients are alive without evidence of disease: ten in first CR ( 7 of 12 Gr I, 1 of 5 Gr II, 2 of 8 Gr III), three in second CR after LR (two Gr I, one Gr II).

Two Gr I patients are alive with evidence of pulmonary metastases.

Nine patients died: six of disease (three Gr II, three Gr III), two because of a second tumor (one Gr I, one $\mathrm{Gr}$ III), and one because of congenital heart disease (Gr III).

One Gr III patient was lost at follow up in CR, 2 years after diagnosis.

The estimated overall survival (OS) and the progression free survival (PFS) rates at 10 years from diagnosis of the 25 patients, calculated with the Kaplan-Meier method, are 67.4 (C.I. 95\%:49-86) and 52.2\% (C.I. 95\%: 31.2-73.2), respectively. Table III summarizes the OS rates of the patients according to age (under vs. over 2 years), initial surgical radicality (IRS Gr I vs. II and III) site of tumors (extremity vs. axial) and protocol (RMS-79 vs. RMS-88). The patients with initial radical excision and those registered in RMS-88 study had a statistically better outcome while the patients under 2 years of age and those with primary localized on extremities had a slightly more favorable prognosis.

The outcome was not influenced with certainty by $\mathrm{T}$ status ( 4 of 15 and 5 of 10 patients with T1 or T2 tumor respectively DOD) and tumor size (4 of 11 and 5 of 9 patients with tumor size $<$ or $>5 \mathrm{~cm}$, respectively DOD).

\section{DISCUSSION}

Fibrosarcoma is a STS of adult and pediatric age, but in infants and young children it is generally considered a separate entity because of its markedly different behavior.

In pediatric age, the clinical features are sligthly different in relation to age. In infancy, fibrosarcomas are found mostly in superficial or deep tissues of distal extremities, while they are axial only in $30 \%$ of cases [1]; proximal regions of extremities, deep trunk and cavitary sites are the favorite localizations in older patients $[1,4]$. This is in agreement with the findings in our series: 10 of 14 children aged $<2$ years had the tumor on extremities (5 in distal segments) and 4 on axial sites, while among 11 patients $>2$ years the tumor was axial in 7 and on extremities in 4 ( 2 distal).

A painless, rapidly enlarging, and dome shaped mass with the overlying skin red, tense, and ulcerated, giving the impression of a vascular tumor, is the main symptom in childhood. The mean diameter of the tumor may exceed $10-15 \mathrm{~cm}$. In older patients, the fibrosarcoma grows slowly and pain is present in one third of the cases [6].

Infantile and adult fibrosarcoma are histologically identical. There are no pathological pictures that allow reliable prediction of the clinical course of the disease $[3,5]$. Histologic grading, a predictor of tumor aggressiveness in adults, has not been proved to be an indicative factor in pediatric age [3,7]. Moreover immunohistochemical and ultrastructural analyses can not identify the two forms. Chromosomal abnormalities have been reported in cases of infantile fibrosarcoma: trisomy 11, deletion of long arm of 17 , and a [12,13] translocation; clonal translocations, translocations [2,15] and [7,22], have also been described in adult-type fibrosarcoma. In our study, the cases were observed before cytogenetic studies became a part of the full examination. At present, a biological evaluation, including cytogenetics, ploidy, and DNA studies, might represent a potentially useful investigation to differentiate the infantile fibrosarcoma from the adult one, so as to improve our knwledge on their clinical behavior $[6,8,9]$.

Despite their common histological aspect and slightly different characteristics at presentation, the two types of fibrosarcomas show different clinical behavior. Infantile fibrosarcoma has an initial rapid growth but an indolent evolution in most instances, so that local recurrence is common but metastatic spread is rare and survival is greater than $80 \%$, especially for tumors originating on the extremities $[2,6,10,11]$. Spontaneous regression of congenital fibrosarcomas has been reported [12] and some authors believe that it should be considered an intermediate form between fibromatosis and adult fibrosarcoma $[4,13]$. In older children and in adolescents, fibrosarcomas tend to behave more like to their adult counterparts: in these cases LR often precedes dissemination and the survival rate is less than $60 \%[1,4,5]$.

The goal of treatment for both infantile and adult fibrosarcoma is the complete nonmutilating excision of the tumor mass [3,6]. An initial radical removal is correlated with the site and the size of the tumor; and is linked to the best prognosis: in our series 11 of $12 \mathrm{Gr}$ I patients are alive ( 9 in $\mathrm{CR}$ and 2 with disease). The 
radicality of the excision at diagnosis was the only significant prognostic factor in our analysis (Table III).

The resection with microscopic residuals does not assure the achievement of the cure. In cases with infantile fibrosarcoma, CT might be recommended if a re-excision is not feasible, while RT is rarely delivered. Considering the low aggressivity of this form some authors recommend only a strict follow-up. The benefit of CT was not evident in our experience. Those two patients treated with surgery alone are alive without disease (one after LR treated with CT and mutilating surgery) while the other two, treated also with CT, had an unfavorable outcome. In patients with inoperable infantile fibrosarcoma, several reports describe good response to $\mathrm{CT}$, adopting different regimens: vincristin and dactinomycin are the recommended drugs in some cases associated with ifosfamide or cyclofosfamide [14-18]. Our data do not support these experiences: in our group the two Gr III patients aged $<2$ years, who received neoadjuvant $\mathrm{CT}$, showed a poor response to VAC as well as to VAIA regimens.

In older patients, an aggressive excision should be the treatment of choice: RT is mandatory when adequate margins can not be obtained and a reoperation is not possible [1,19]. CT is indicated preoperatively in cases of inoperable tumors, even if its efficacy is still debated, and postoperatively because of the frequent occurrence of micrometastases [20,21]. In our experience, 10 of 11 patients had CT: no data are available concerning its efficacy in Gr I-II. Three out of five Gr III cases were treated with VAC achieving a partial response, while two treated with VAIA showed a minor response. Among these five patients those two who underwent radical surgery and one of the three treated with RT achieved the CR.

The use of the term infantile type rather than adult type of fibrosarcoma is highly influenced by the age of the patient. In the absence of other tools, age is a crucial point in planning the treatment and defining the outcome of the disease. Many cases of fibrosarcoma are diagnosed in the first year of life and 50\% of them are congenital. Some authors consider as infantile fibrosarcomas those forms diagnosed before $4-5$ years of age $[5,10,11,13]$, while others accept only those cases within 2 years [3,22]. In a group of 12 neonatal fibrosarcomas, the outcome was favorable in all the patients: 10 had had a complete surgery and only 1 received postoperative CT [23].

On the other hand, patients of intermediate age (3-6 years) remain a therapeutic challenge for oncologists, surgeons, and radiotherapists.

In our group, the 14 patients under 2 years of age $(11<1$ year and 7 of them congenital) had a better outcome than the older ones. Most of these tumors were not invasive and could be excised completely at diagnosis: 11 of 14 are alive without disease (5 of 7 among the congenital forms), 2 are DOD and 1 died because of cardiac malformation; in particular none of 10 patients with a primary tumor on extremities DOD. LR was the main cause of treatment failure, but 3 of 4 relapsed patients were cured with further multimodal therapy.

Among the 11 patients aged $>2$ years, 5 achieved first CR (but 2 died of second tumor), 2 are alive with disease after local and distant relapse, 4 DOD following LR and metastatic spread. We must underline that none of the patients were cured after LR or pulmonary metastases. The outcome of the three patients aged between 2 and 5 years, whose forms could be considered infantile, was similar to that of the older patients. Only one is alive in first CR after multimodal intensive treatment, one is alive with disease after LR and pulmonary metastases, while one patient died of PD.

Nowadays mutilating surgery is recommended only in case of poor response after primary CT or in case of relapse, for infantile as well as for adult fibrosarcoma. In our group, 4 of 25 patients had a mutilating excision. It was performed initially in one case who achieved the CR, at delayed surgery in one adolescent achieving the CR, and after LR in other two patients (only one infant obtained the CR). At present, we would avoid initial mutilating surgery and start with neoadjuvant therapy.

\section{CONCLUSIONS}

The results achieved in our series are similar to those reported in the literature $[3,5,17]$. The significantly better OS registered in the patients of the RMS- 88 protocol is probably related to the high number of complete resection at diagnosis $(n=9)$ and the high rate of patients $<2$ years registered in this study $(\mathrm{n}=10)$.

An initial complete excision was the treatment of choice and was related to the best outcome, being the only significant prognostic factor. The presence of microscopical residuals after primary surgery caused a LR in 2 of 4 patients: CT was not effective in these patients. Neoadjuvant CT determined a partial response in two cases, in particular no conclusions are possible in this series concerning the efficacy of CT for infantile fibrosarcomas, as reported in previous experiences.

Patients under 2 years of age had a better prognosis than the older ones; however, this favorable outcome was influenced by the tumor site and the surgical treatment. In fact, most of them had a tumor on extremities that could be excised at diagnosis. Moreover, most of the infantile fibrosarcomas with LR were cured by aggressive therapy (including mutilating surgery). In older patients, a multimodal treatment is required as in adults: in our group all the patients who relapsed had an unfavorable outcome. 


\section{ACKNOWLEDGEMENTS}

The Authors thank all the participants to the study and Dr. Lucia Masiero for statistical assistance.

\section{REFERENCES}

1. Miser JS, Triche TJ, Kinsella TJ, et al.: Other soft sarcomas in children. Chapter 32. In: Pizzo PA, Poplack DG (eds): "Principles and Practice of Pediatric Oncology." 3rd edn. Philadelphia: Lippincott-Raven, 1997:873-876.

2. Carli M, Guglielmi M, Sotti G, et al.: Soft tissue sarcomas. Chapter 15. In: Pinkerton CR, Plowman PN (eds): "Paediatric Oncology, Clinical Practice and Controversies." 2nd edn. London: Chapman \& Hall Medical, 1997:380-416.

3. Coffin CM: Congenital-Infantile fibrosarcoma: a comparison with adult-type fibrosarcoma. In: Coffin CM, Dehner LP, O'Shea PA (eds): "Pediatric Soft Tissue Tumors. A Clinical, Pathological, and Therapeutic approach." Baltimore: Lippincott 1997:164-170.

4. Coffin CM, Jaszcz W, O'Shea P, et al.: So-called congenitalinfantile fibrosarcoma: does it exist and what is it? Pediatr Pathol 1994;14:133-150.

5. Enzinger FM, Meisss W: Fibrosarcoma. In: "Soft tissue tumors." 3rd edn. St.Louis: Mosby-Year Book, 1995:269-291.

6. Fisher C. Fibromatosis and fibrosarcoma in infancy and childhood. Eur J Cancer 1996;32A(12):2094-2100.

7. Scott SM, Reiman HM, Pritchard DJ, et al.: Soft tissue fibrosarcoma. A clinicopathologic study of 132 cases. Cancer 1989;64:925-931.

8. Ladenstein R, Strehl S, Ambros P, et al.: Translocation $(12,13)$ in a case of poor prognosis infantile fibrosarcoma. Abstracts SIOP XXIII Meeting. Med Pediatr Oncol 1991;19(5):378-384.

9. Sankary S, Dickman PS, Wiener E, et al.: Consistent numerical chromosome aberrations in congenital fibrosarcoma. Cancer Genet Cytogenet 1993;65:152-156.

10. Soule EH, Pritchard DJ: Fibrosarcoma in infants and children Cancer 1977;40:1711-1721.
11. Corsi A, Boldrini R, Bosman C: Congenital-Infantile fibrosarcoma: study of two cases and review of the literature. Tumori 1994;80:392-400.

12. Madden NP, Spicer D, Allibone EB, et al.: Spontaneous regression of neonatal fibrosarcoma. Br J Cancer 1992;66 (Suppl XVIII):572-575.

13. Chung EB, Enzinger FM: Infantile fibrosarcoma. Cancer 1976;38:729-739.

14. Ninane J. Chemotherapy for infantile fibrosarcoma: letter to the editor. Med Pediatr Oncol 1991;19:209.

15. Desbois JC, Delepine N, Cornille H, et al.: Congenital fibrosarcoma: a case for a neoadjuvant chemotherapy. Abstracts SIOP XXIII Meeting. Med Pediatr Oncol 1991;19(5):366.

16. Kinaston JA, Malcom AJ, Craft AW: Chemotherapy in the management of infantile fibrosarcoma. Abstracts SIOP XXIII Meeting. Med Pediatr Oncol 1991;19(5):378.

17. Sommelet D, Oberlin O, Terrier-Lacombe MJ, et al.: "Fibrosarcomas in childhood." Third Int. Soft Tissue Sarcoma Congress, Stuttgart 1997. Book of Abstracts. 1997:p 70.

18. Shetty AK, Yu LC, Gardner RV, et al.: Role of chemotherapy in the treatment of infantile fibrosarcoma. Med Pediatr Oncol 1999;33:425-427.

19. Greager JA, Reichard K, Campana JP, et al: Fibrosarcoma of the head and the Neck. Am J Surg 1994;167:437-439.

20. Rosemberg JA, Tepper J, Glotstein T, et al.: Prospective randomize evaluation of adjuvant chemotherapy in adults with STS of the extremities. Cancer 1983;52:424-429.

21. Miser JS, Kinsella TJ, Triche TJ, et al.: Treatment of recurrent childhood sarcomas and primitive neural tumors with ifosfamide, etoposide and mesna. J Clin Oncol 1987;5:11911193.

22. Salloum E, Caillaud JM, Flamant F, et al.: Poor prognosis infantile fibrosarcoma with pathologic features of malignant fibrous histiocytoma after local recurrence. Med Pediatr Oncol 1990;18:295-298.

23. Dillon PW, Whalen TV, Azizkhan RG, et al.: Neonatal soft tissue sarcomas: the influence of pathology on treatment and survival. J Pediatr Surg 1995;30(7):1038-1041. 